\title{
An Unusual Case of Retro-Orbital Pain with Diplopia
}

\author{
Michael Paci, Theodore H. Wein, Sabah Bekhor
}

Can. J. Neurol. Sci. 2010; 37: 888-889

Tolosa-Hunt syndrome (THS) is an idiopathic inflammatory process that involves the cavernous sinus and/or the superior orbital fissure and manifests with symptoms of ophthalmoplegia and headache ${ }^{1}$. Tolosa-Hunt syndrome has an estimated incidence of one to two cases per million ${ }^{2}$. Criteria for diagnosis of THS as set by the International Headache Society (IHS) in 2004 include (1) one or more episodes of unilateral pain persisting for weeks if untreated, (2) paresis of one or more of the third, fourth and/or sixth cranial nerves and/or demonstration of granuloma by magnetic resonance imaging (MRI) or biopsy, (3) paresis coinciding with the onset of pain or following it within two weeks, (4) pain and paresis resolving within 72 hours when treated adequately with corticosteroids, (5) other causes have been excluded by appropriate investigations ${ }^{3}$.

Tolosa-Hunt syndrome is a benign process during which infiltration of lymphocytes and macrophages into the septae and walls of the cavernous sinus and / or the superior orbital fissure leads to a primarily granulomatous inflammatory reaction which clinically leads to facial pain ${ }^{1}$. The inflammation can in turn affect nerves passing through the cavervous sinus and/or superior orbital fissure. The oculomotor, abducens and trochlear nerves, as well as the ophthalmic branch of the trigeminal nerve, are most commonly involved. As many processes can involve the cavernous sinus and the superior orbital fissure, differential diagnoses may includes: trauma, tumour, cavernous sinus thrombosis, infectious disease, vasculitis, sarcoidosis, diabetes mellitus and ophthalmoplegic migraine ${ }^{1,3}$.

Controversy surrounding THS is two-fold. First, can a diagnosis of THS be given without evidence of granuloma on MRI or biopsy ${ }^{4}$ ? The IHS guidelines are unclear on this point. Some, such as La Mantia et $\mathrm{al}^{4}$, interpret the second criterion in a strict manner, i.e. that the IHS requires evidence of granuloma on either MRI or biopsy in order for a true diagnosis to be made. Others, such as Colnaghi et $\mathrm{al}^{5}$, interpret the second criterion more loosely and consider that the it can be filled by clinical demonstration of paresis of the third, fourth and/or sixth cranial nerves.

Second, must THS always be a diagnosis of exclusion ${ }^{6,7}$ ? We present a case of unusual diplopia with retro-orbital pain which we believe underlines the importance of these two clinical questions.

\section{CASe}

A 76-year-old right-handed female presented with a twoweek history of right retro-orbital pain accompanied by a oneweek history of horizontal diplopia. The patient reported difficulty walking up stairs due to her diplopia. She denied any jaw claudication, weakness, paresthesia, nausea, vomiting, photosensitivity, loss of consciousness, trauma or history of thrombosis. Her past medical history was remarkable for hypertension and osteoarthritis treated with telmisartan/ hydrochlorothiazide and acetaminophen.

On examination, the patient was alert and oriented. Ptosis of the right eyelid was evident and extra-ocular movement exam revealed paresis of the right oculomotor, abducens and trochlear nerves. Pupils were round and equally responsive to light. Fundoscopic exam revealed sharp disc margins and normal venous pulsation. Sensory exam revealed decreased sensation in the territory of the ophthalmic branch of the right trigeminal nerve with a decreased right corneal reflex. Except for a mildly ataxic gait, the remainder of the neurological exam was unremarkable.

An MRI with gadolinium as well as a magnetic resonance angiography of the circle of Willis were unremarkable as was an echocardiogram. A lumbar puncture (LP) revealed normal opening pressure, no leukocytosis, normal levels of protein and glucose. Culture of the cerebro-spinal fluid obtained grew no organisms. A right temporal artery biopsy was unremarkable. Serologies revealed a normal glucose level, a c-reactive protein level of $3.5 \mathrm{mg} / \mathrm{L}$ and an erythrocyte sedimentation rate of 18 $\mathrm{mm} / \mathrm{h}$. The blood work was negative for anti-neutrophil cytoplasmic antibodies and revealed normal levels of angiotensin converting enzyme.

A course of $50 \mathrm{mg}$ of prednisone daily was prescribed. There was remarkable improvement within 72 hours, with resolution of the headache and near complete resolution of the sensory loss. The patient was discharged on the fourth day with mild horizontal diplopia. At a follow-up seven days after the start of treatment, all symptoms had resolved. The patient remained on $50 \mathrm{mg}$ of prednisone daily for five weeks, after which the dose was slowly tapered over two weeks. At the fifth week of treatment, the patient consulted a neuro-ophthalmologist for symptoms of jaw claudication and headache. New serology revealed an elevated c-reactive protein level of $20 \mathrm{mg} / \mathrm{L}$, however her erythrocyte sedimentation rate was normal. A unilateral temporal artery biopsy was normal. These new

From the Departments of Neurology and Neurosurgery (THW, SB), St. Mary's Hospital, McGill Medical School (MP), McGill University, Montreal, Quebec, Canada. Received March 10, 2010. Final Revisions Submitted June 22, 2010. Correspondence to: Theodore H. Wein, Division of Neurology, St. Mary's Hospital, 3830 Lacombe, B234, Montreal, Quebec, H3T 1M5, Canada. 
symptoms resolved before the end of the prednisone treatment two weeks later. At a follow-up appointment with her neuroophthalmologist five months after the onset of the first symptoms of THS, the patient remained entirely asymptomatic.

\section{Comments}

Our case underlines the difficulty of obtaining a diagnosis of THS according to the 2004 IHS guidelines for certain patients. In fact, our case answered all the IHS criteria for THS except for evidence of granuloma on MRI or biopsy. Our patient's MRI was unremarkable. Although most cases of suspected THS syndrome have enlargement of the cavernous sinus on MRI, some cases were found to show changes only on repeat examination ${ }^{8,9}$. Kobor et al found that of 38 reviewed cases of THS reporting imaging results, eight initially had unremarkable MRI findings, three of which later had positive findings on repeat imaging ${ }^{8}$. In our case, MRI was not repeated. La Mantia et al reported that 41 out of 124 cases of THS reviewed had no evidence of inflammation on MRI or biopsy ${ }^{4}$. The question remains as to whether patients with suspected THS should all undergo a repeat MRI examination shortly after the first if the later is unremarkable in order to confirm the diagnosis. This also leads to the question of cavernous sinus biopsy. Such a procedure has significant risks, even when guided by computed tomogram (CT), which include damage to the structures within the sinus, including the cranial nerves and the carotid artery, as well as bleeding ${ }^{10}$. The risks associated with such a procedure may not outweigh the benefit of a positive biopsy result for granuloma in a case with an unremarkable MRI, in which all other clinical data and investigations point towards THS.

The controversy of whether THS should be a diagnosis of exclusion is also emphasized by our case ${ }^{6,7}$. We certainly thought that it should, which is why we undertook many investigations to exclude other potential causes. Rousseaux et al suggest that positive findings MRI, in a case with all other clinical data and investigations pointing to THS, may not always seal the diagnosis, as some early malignant and infectious processes could appear similarly ${ }^{6}$. They conclude that MRI findings may not be sufficient to consider THS as a diagnosis of inclusion. Kwan et al underline that many processes respond to steroids, and that this criterion does not allow THS to be considered a diagnosis of inclusion ${ }^{7}$. However, they also suggested that with better imaging techniques, THS could eventually become a diagnosis of inclusion. This seems unlikely, as better imaging may still not allow for proper categorization of those who, although all findings point toward THS, have an initial unremarkable MRI.

Our case underlines the question of whether MRI or biopsy evidence of granuloma is truly needed to make a diagnosis of THS when there is very strong clinical suspicion and all other IHS criteria are met. It also underlines the fact that THS may never become a diagnosis of inclusion based on imaging criteria.

\section{REFERENCES}

1. Kline LB, Hoyt WF. Nosological entities?: the Tolosa-Hunt Syndrome. J Neurol Neurosurg Psychiatry. 2001;71:577-82.

2. Iaconetta G, Stella L, Esposito M, Cappabianca P. Tolosa-Hunt syndrome extending in the cerebello-pontine angle. Cephalalgia. 2005;25:746-50.

3. Headache Classification Sub-Committee of the International Headache Society. The International Classification of Headache Disorder, 2nd ed. Cephalalgia. 2004;24(1):1-160.

4. La Mantia L, Curone M, Rapoport AM, Bussone G. Tolosa-Hunt syndrome: critical literature review based on IHS 2004 criteria. Cephalalgia. 2006;26:772-81.

5. Colnaghi S, Versino M, Marchioni E, et al. ICHD-II diagnostic criteria for Tolosa-Hunt syndrome in idiopathic inflammatory syndromes of the orbit and/or the cavernous sinus. Cephalalgia. 2008;28:577-84

6. Rousseaux P, Peruzzi P, Schaison-Cusin M. Le syndrome de TolosaHunt doit-il encore être un diagnostic d'élimination? (French, Tolosa Hunt syndrome: still a diagnosis by exclusion?) Rev Neurol. 1993;149:315-19.

7. Kwan ESK, Wolpert SM, Hedges TR III, Laucella M. Tolosa-Hunt syndrome revisited: not necessarily a diagnosis of exclusion. Am J Roentgenol. 1988;150:413-18.

8. Kobor J, Voros E, Deak A. Magnetic resonance imaging in TolosaHunt syndrome. Eur J Pediatr. 2004;163:753-4.

9. Schuknecht B, Sturm V, Huisman TAGM, Landau K. Tolosa-Hunt syndrome: MR imaging features in 15 patients with 20 episodes of painful ophthalmoplegia. Eur J Radiol. 2009;69:445-53.

10. Yi W, Ohman K, Brannstrom T, Bergenheim AT. Percutaneous biopsy of cavernous sinus tumour via the foramen ovale. Acta Neurochir. 2009;151:401-7. 